\title{
A Study to Assess Whether Planned Pre-Operative Education Decreases Anxiety and Improves Satisfaction in Patients Undergoing Surgery-A Randomized Controlled Trial
}

\author{
Vishnu Priya. ${ }^{1}$, Sumi Mariea Sebastian ${ }^{2}$, Miriam George Fenn ${ }^{3}$, \\ Anna Mathew ${ }^{4}$, Saravana Kumar Velusamy ${ }^{5}$ \\ ${ }^{1}$ IIIyear MBBS, MOSC Medical College Hospital, Kolenchery, Kerala, India \\ ${ }^{2}$ III year MBBS, MOSC Medical College Hospital, Kolenchery, Kerala, India \\ ${ }^{3}$ Associate Professor, Department of Obstetrics And Gynaecology, MOSC Medical College Hospital, \\ Kolenchery, Kerala, India \\ ${ }^{4}$ Research Co-ordinator\& Professor, Department of Pharmacology, MOSC Medical College Hospital, \\ Kolenchery,, Kerala,India \\ ${ }^{5}$ Biostatistician, Department of Biostatistics, Christian Medical College, Vellore, Tamil Nadu, India.
}

\begin{abstract}
:
Background: Patients preparing for surgery are invariably anxious and apprehensive. Studies have shown that planned pre-operative education significantly reduces anxiety and improves satisfaction.

Objectives: To assess whether planned pre-operative education decreases anxiety and improves satisfaction in patients undergoing uterine fibroid surgery.

Methodology: 64 patients admitted for fibroid surgery were randomized, to the intervention group $(n=32)$ which received planned pre-operative education and to the control group $(n=32)$ which didnot receive education. The anxiety levels of patients in both the groups were assessed prior to giving education using APAIS (Amsterdam Pre-operative Anxiety and Information Scale) and were reassessed by a blinded assessor after giving education on the day before surgery. The patient satisfaction was measured using PSQ-18 (Patient Satisfaction Questionnaire short form) on the second day after surgery.

Results: The mean combined anxiety score of APAIS showed a significant decrease in the intervention group, after giving education. All three subscales of APAIS also showed a decrease in the educated group. The overall patient satisfaction score and all seven subscales of PSQ-18 showed a significant increase in the intervention group.

Conclusion: The study highlights the fact that planned pre-operative education can have a significant impact on anxiety and satisfaction of patients.
\end{abstract}

Keywords: planned pre-operative education, anxiety, satisfaction, APAIS, PSQ-18.

\section{Introduction}

Patients preparing for surgery are invariably anxious and apprehensive. Studies have demonstrated that pre-operative patient education is highly beneficial as it helps in achieving emotional inoculation. ${ }^{1}$ It has also been shown that pre-operative education has a positive impact on psychological well-being, anxiety and satisfaction of patients. ${ }^{2}$ However,contrary to this,Shuldham showed that many studies were unable to prove a beneficial effect because of small sample size, ambiguity in randomization protocols, dearth of blinding and uniformity of samples. ${ }^{3}$ In a similar study conducted by the same investigator in patients undergoing coronary artery bypass surgery no remarkable effect of pre-operative education on anxiety,pain or depression was found. ${ }^{4,5}$ In summary the impact of pre-operative education on ameliorating patients'understanding,mitigating their anxiety and increasing satisfaction remains uncertain. The aim of this study is to find out if planned preoperative education using principles of effective communication develops a positive impact on the attitude of patients.For the purpose of limiting bias we have restricted our participants to those admitted for fibroid surgery. Fibroids are the commonest tumours which occur in $20 \%$ of all women who are in the reproductive age group. ${ }^{6}$ In this study, two valid scales were used to assess the anxiety and measure the satisfaction.The preoperative anxiety level of patients and the need for information were assessed using The Amsterdam Preoperative Anxiety and Information Scale(APAIS) and patient satisfaction was measured using the Patient Satisfaction Questionnaire Short form(PSQ-18) which has become a vital component of health care outcomes. ${ }^{7,8}$ 


\section{Materials and Methods}

2.1. Type of study: This is a randomized, parallel group, double blinded clinical trial. This study was approved by the institutional review board and received ethics committee approval from the institutional ethics committee .The study has been registered with the Clinical Trials Registry-India (CTRI). The trial No. CTRI/2014/06/004649.

2.2. Participants: All patients hospitalized for fibroid surgery in MOSC Medical College Hospital who have given informed consent and fulfilled the inclusion criteria.

2.3. Inclusion criteria: All women between $30-55 \mathrm{yrs}$ of age admitted for fibroid surgery in the gynaecology ward of the hospital.

2.4. Exclusion Criteria: All medical professionals admitted for fibroid surgery, patients with psychiatric illness.

2.5. Sample size calculation: The sample size was calculated using the nMaster Sample Size Calculation Software (produced by the Department of Biostatistics, Christian Medical College, Vellore) for the nonparametric two group Wilcoxon Mann Whitney U-Test.The sample size was found to be 32 in each group(X, Y) with a power of $80 \%$ and an alpha error of $5 \%$. The probability that a score from $\mathrm{X}$ is larger than a score from $\mathrm{Y}$ is larger than $1 / 2$ is taken to be 0.7 . Thus 32 subjects were recruited into each arm of the study.

2.6. Sampling technique: Participants were randomized into two groups - Group $X$ and Group $Y$ using fixed, permuted block randomization using sequential blocks. ${ }^{9}$ Blocks of four having equal numbers of $\mathrm{X}$ and $\mathrm{Y}$ were used. With the block size of four for groups $\mathrm{X}$ and $\mathrm{Y}$, the six possible permutations were coded as XXYY, XYXY, XYYX, YXXY, YXYX and YYXX. The random sequence selected the next block, determining the next four participant allocation (ignoring numbers $0,7,8,9$ ).

2.7. Instruments: The two instruments used in the study are The Amsterdam Pre-operative anxiety and Information Scale (APAIS) and Patient Satisfaction Questionnaire Short form (PSQ-18).APAIS is applied by the investigator prior to education and a blinded assessor repeated the same in both the groups after the education on the day before surgery. This is a six-item questionnaire. It consists of two questions each assessing the patients' anxiety regarding surgery and anaesthesia and two questions assessing the need for information. It is divided into 4 components-Sum A (Anaesthesia related anxiety), Sum S (Surgery related anxiety), Sum C (Combined anxiety component=SumA+SumS), Sum I (Information desire component). Patient Satisfaction Questionnaire Short form (PSQ-18) is applied by the investigator in both the groups on the second day after the surgery. This is an eighteen item questionnaire and it has seven subscales assessing general satisfaction, technical quality, interpersonal manner,communication,financial aspects, time spent with doctor, accessibility and convenience.

2.8. Study procedure: This study is a randomized, parallel group clinical trial. Patients admitted for fibroid surgery in the gynaecology ward of the hospital were recruited if they fulfilled the inclusion criteria and gave informed consent and were randomized into two groups by permuted block randomization. Group X(Intervention group) received planned pre-operative education in addition to the routine counselling given by the treating doctor and Group Y-(Control Group) received only the routine counselling from the treating doctor. The primary outcome was the assessment of anxiety using APAIS and the secondary outcome was the satisfaction measurement using PSQ-18.The primary outcome was assessed by the investigator in the intervention group before giving the education and also in the control group (APAIS-1). After giving education it was repeated in both the groups by a second assessor (APAIS-2).This was done on the day before the surgery. The secondary outcome was assessed by the investigator on the $2^{\text {nd }}$ day after the surgery in both the groups..The results were collated and statistically analysed. This is a double blinded trial where both the participant and the second assessor who conducts the APAIS-2 are blinded. The blinding has been used to decrease the bias and to achieve comparability between the two groups.

2.9. Intervention: The intervention used in this study is planned pre-operative education. It includes a teaching session and an interactive session.

2.9.1. Teaching session: In this study the planned pre-operative education was systematically delivered to study group using the method of verbal communication using good communication skills. It included bedside meeting with the patient, obtaining the informed consent and establishing a good rapport with them by taking a clinical 
history, and explaining them about the surgical procedure and anaesthesia in simple language and giving them assurance regarding the competence of doctors.

2.9.1.1. Information regarding the cause,symptoms,prevalence,progression and recurrence:The patients were informed that the actual etiology is unknown but the possible causes are due to the ovarian steroid hormones, and the risk factors -early age at menarche,nulliparity,obesity.The common symptoms are irregular bleeding,heavy bleeding during periods,pelvic pain,constipation,frequent urination/difficulty in emptying bladder,backache,pain during sexual intercourse. Uterine fibroids constitute about $6.8 \%$ of the gynaecological admission in India.There is only $0.1 \%$ chance of malignancy in fibroids .Hysterectomy offers a true cure and eliminates the chance of recurrence.

\subsubsection{Types of surgery: The common surgical procedures which are used include}

2.9.1.2.1. Myomectomy: In this surgery only the fibroids are removed and leaves the uterus intact and so fertility is retained.But since there is fairly high incidence of regrowth, this is not preferred in women who have completed their family.

2.9.1.2.2. Hysterectomy: It involves removal of uterus and is of three different types-total abdominal hysterectomy,vaginal hysterectomy and laparoscopy assisted vaginal hysterectomy.Total abdominal hysterectomy is done through a tranverse incision made through the abdominal wall.The advantages are that the uterus can be removed even if a woman has internal scarring from previous surgery or if the fibroids are large.The surgeon has a good view of the abdominal cavity and it allows the whole abdomen and pelvis to be examined.Vaginal hysterectomy is a procedure where the surgeon makes an incision inside and near the top of the vagina.The surgeon then reaches the uterus through this incision ,and once the it is cut free,it is removed through the vagina.The advantages are that it leaves no visible scar and is less painful and usually has faster recovery.Laparoscopy assisted vaginal hysterectomy is performed using small incisions made in the abdomen through which slender metal tubes (trocars) are inserted which provide passage for a laparoscope(which is like a tiny telescope) and other microsurgical tools. After detaching from the surrounding structures, uterus is removed through an incision made in the vagina.The advantages are that the scars are very small and the pain and recovery time are less.

2.9.1.3. Assurance is given to the patients regarding the efficiency of the doctors.

2.9.1.4. Preparations before surgery: The patients were given information regarding the preparations to be done on the night before surgery(including the reasons for fasting prior to surgery),preparations on the day of the surgery(removing jewellery,dentures, wearing hospital gown and taking medications)..

2.9.1.5. Anaesthesia: The type of anaesthesia, mode of administration, sequelae and the fact that there was no reason for anxiety were explained.

2.9.2. Interactive session: The interactive session involved active listening to the queries of the patients and clearing their doubts .The questions asked by majority of the patients were regarding the post-operative care, duration of hospital stay, complications following surgery and the expected activities after surgery.The information given was able to tackle their queries and make them mentally prepared for the surgery.

2.9.2.1.Post-operative care: Patients were given information regarding the post- operative care which includes shifting to the recovery room,then to surgical ICU where they will be managed using i.v fluids ,catheter will be inserted to drain urine and once the condition is stable ,shifting to the ward and they were assured that postoperative pain will be managed using analgesics.

2.9.2.2. Hospital Stay: Hysterectomy usually requires three to five days of stay in hospital.

2.9.2.3. Complications following surgery: Patients were assured that in the unlikely event of complications arising during surgery such as bleeding, infections, injury to structures near the uterus, the surgeons are competent to manage the situation. The problem of early menopause can be easily managed.

2.9.2.4. Expected activities after the surgery: As early mobilisation is the dictum following surgery, patients were encouraged to take small walks following the surgery in a graded manner. They were advised to take 
adequate rest for six weeks and not to lift any heavy weights during this period.Information regarding the follow-up and also about the pelvic floor exercises were given.

3.0. Ethical Considerations: Approval was obtained from the Institutional Review Board and Ethics Committee of MOSC Medical College Hospital. Each of the participants was given an information sheet explaining the purpose of the study and details of the procedure. They were given the opportunity to ask questions and clarify their doubts regarding participation.Then a consent form was given whereby their voluntary participation in the study was ensured.All data collected in this study is promised to be kept confidential and used only for scientific purpose.

3.1. Analysis of data: The data collected was entered into an Excel spreadsheet, and then converted into SPSS, version 16 for further analysis. All continuous variables were checked for normal distribution.The continuous variables which satisfied normal distribution assessment were given using mean and standard deviation and for the variables which did not satisfy this condition, median and interquartile range were taken.Outcome measure which satisfied normal distribution was assessed using Independent Sample t-test and those which did not satisfy normal distribution were assessed using non parametric Wilcoxon Mann-Whitney U-Test.p value $<0.05$ was taken to be significant and $<0.001$ was taken to be highly significant.

\section{Results}

Fig 1 shows the study flow chart illustrating the study procedure. The baseline demographic data and baseline results of questionnaires of the patients is presented in Table 1.The mean age in Group X was found to be $45.59 \pm 2.781 .40 .6 \%$ in Group $X$ belonged to low, $37.5 \%$ to middle and $21.9 \%$ to high socioeconomic status respectively.43.8\% had school education, 50\% were graduates, $6.2 \%$ were post-graduates and $28.1 \%$ of the patients had undergone previous surgeries.

In Group X,the mean APAIS-1(Sum C-1) score was found to be 13.25 \pm 4.879 . Among the subscales, median Sum I-1 score was found to be 8.00,(5.00-9.00),median Sum A-1 and Sum S-1 scores were found to be the same- 7.00(5.00-9.00).

Table 2 illustrates the outcome analysis. In Group X, the mean APAIS-2 (SumC-2) score was found to be 8.06 \pm 4.272 . Among the subscales, median Sum A-2 and Sum S-2 scores were found to be the same-3.00(2.005.00) and median Sum I-2 score was found to be 7.00(5.00-7.75).A significant p value was obtained for the combined anxiety component and the surgery related anxiety component.

In Group X, for PSQ-18,the median overall satisfaction score was found to be 69.00,(48.25-75.00) with highly significant $\mathrm{p}$ value.The median scores for the subcales were found to be general satisfaction $3.50,(3.00-4.00)$ technical quality 3.625,(3.250-4.188) interpersonal manner,4.00,(2.00-4.50),communication 4.00,(3.00-4.50), financial aspects 4.12,(2.38-4.50),time spent with doctor 3.75,(2.50-4.50), accessibility and convenience 3.5000,(2.3125-4.2500). A highly significant $\mathrm{p}$ value was obtained for technical quality, communication, time spent with doctor and accessibility and convenience and significant values were obtained for the remaining subscales.

\section{Discussion}

From the study it was found that the baseline characteristics were found to be comparable in Group X and group $\mathrm{Y}$, indicating effective randomization. Though the number of participants belonging to the high socioeconomic status and the number of post-graduates were marginally higher in Group X than Group Y, further adjustment was not required as the combined anxiety component of APAIS, before giving planned pre-operative education was found to be comparable in both the groups. The number of participants who had undergone previous surgeries was nearly equal in both the groups.

4.1.APAIS: The mean combined anxiety component of APAIS after giving education,was found to be significantly reduced in Group X (8.06 \pm 4.272$)$ as compared to Group $\mathrm{Y}(11.84 \pm 5.052)(\mathrm{p}=0.002)$. Among the other components, the surgery related anxiety was found to be significantly reduced in Group $X(p=0.03)$. So this study has proved the primary objective that giving planned pre-operative education has resulted in a significant decrease in the anxiety of the patients with significant decrease in surgery related anxiety..The anaesthesia related anxiety also decreased in Group X as compared to Group Y but it was not found to be statistically significant.In both the groups,the information desire component showed a marginal reduction but it was also not found to be statistically significant.

The reason why the anxiety levels have decreased points to the effectiveness of the planned preoperative education given to the patients in a systematic manner.In a similar study,assessing the importance of anaesthetist's visit prior to surgery,there was a significant decrease in combined anxiety and anaesthesia related anxiety. ${ }^{10}$ 
4.2.PSQ-18: The patient satisfaction as analysed by the PSQ-18 showed a significant improvement following education. The overall satisfaction was found to be high in Group X(69.00,(48.25-75.00) as compared to Group $\mathrm{Y}(41.50,(31.00-62.50)$ and statistically, it was found to be highly significant(p>0.001).All the subscales of PSQ18 showed a statistically significant increase in GroupX,with technical quality, communication, time spent with doctor,accessibility and convenience being highly significant $(\mathrm{p}<0.001)$. So the study has proved the secondary objective that planned pre-operative education improves the satisfaction of patients.

The reason for the significant increase in the patient satisfaction is the effectiveness of the education, which has taken into account the basic principles of effective communication, and which involved bedside meeting with the patient, active listening and systematic delivery of information with an empathetic attitude.

In a cross-sectional study conducted to assess the patient satisfaction with ophthalmic services, the regression model could predict an overall satisfaction of $60 \%$ with accessibility $(\mathrm{p}<0.001)$ and technical quality $(\mathrm{p}<0.001)$ having the strongest association with the overall satisfaction. ${ }^{11}$

Thus this study has shown that the patient approaches surgery with less anxiety when planned pre-operative education is given. It has also shown that the level of patient satisfaction is much greater following this intervention.

\section{Conclusion}

The study highlights the fact that planned pre-operative education given in a systematic manner using the method of verbal communication using good communication skills can have a significant impact on reducing the anxiety of patients as well as improving their satisfaction.It can bring about substantial changes in the arena of patient care in the future.It also points to the importance of conducting more number of studies in this part of the country so that we can conclusively establish the fact that planned pre-operative education is beneficial to the patients.

\section{Acknowledgements}

I would like to acknowledge the Indian Council of Medical research Short Terrm Studentship program for giving me the opportunity to do this study (Reference ID-2014-01169).I would like to extend my sincere gratitude to the Dean, Medical Superintendent and the administration of MOSC Medical College Hospital, Kolenchery for their encouragement and support and the Department of Obstetrics and Gynaecology for granting me permission to do the study.

\section{References}

[1]. Janis, Irving L, Psychological Stress: Psychoanalytic and behavioural studies of surgical patients( New York, Wiley, 1958).

[2]. Devine, E. C,Effects of psychoeducational care for adult surgical patients: a meta-analysis of 191 studies, Patient Education and Counseling, 19(2),1992,129-142.

[3]. Shuldham CM, Pre-operative education - a review of the research design, International Journal of Nursing Studies, 36(2), 1992, 179-187.

[4]. Shuldham CM,Pre-operative education for the patient having coronary artery bypass surgery, Patient Education and Counseling, 43(2),2001,129-137.

[5]. Shuldham CM, Fleming S, Goodman H.,The impact of pre-operative education on recovery following coronary artery bypass surgery. A randomized controlled clinical trial, European Heart Journal,23(8),2002,666-674.

[6]. Briggs ND, Common gynaecological tumours, Tropical Journal of Obstetrics andGynaecology,12(12),1995,62-71.

[7]. Moerman N,van Dam FS,Muller MJ,Oosting H,The Amsterdam Pre-operative Anxiety and Information Scale(APAIS),Anesthesia \& Analgesia, 82(3),1996,445-451.

[8]. Marshall GN,Hays RD,The Patient Satisfaction Questionnaire Short Form(PSQ-18)(Santa Monica,CA,RAND Corporation,1999,P7865).

[9]. Matts J, Lachin J,Properties of permuted-block randomization in clinical trials, Controlled Clinical Trials,9,1988,327-344.

[10]. Matthias AT,Samarasekera DN,Preoperative anxiety in surgical patients-experience of a single unit, Acta Anaesthesiologica Taiwanica,50(1),2012,3-6.

[11]. Ziaei H, Katibeh M, Eskandari A, Mirzadeh M, Rabbanikhah Z, Javadi MA.,Determinants of patient satisfaction with ophthalmic services. BMC Research Notes,4(7),2011 
Figure 1.Study flow chart

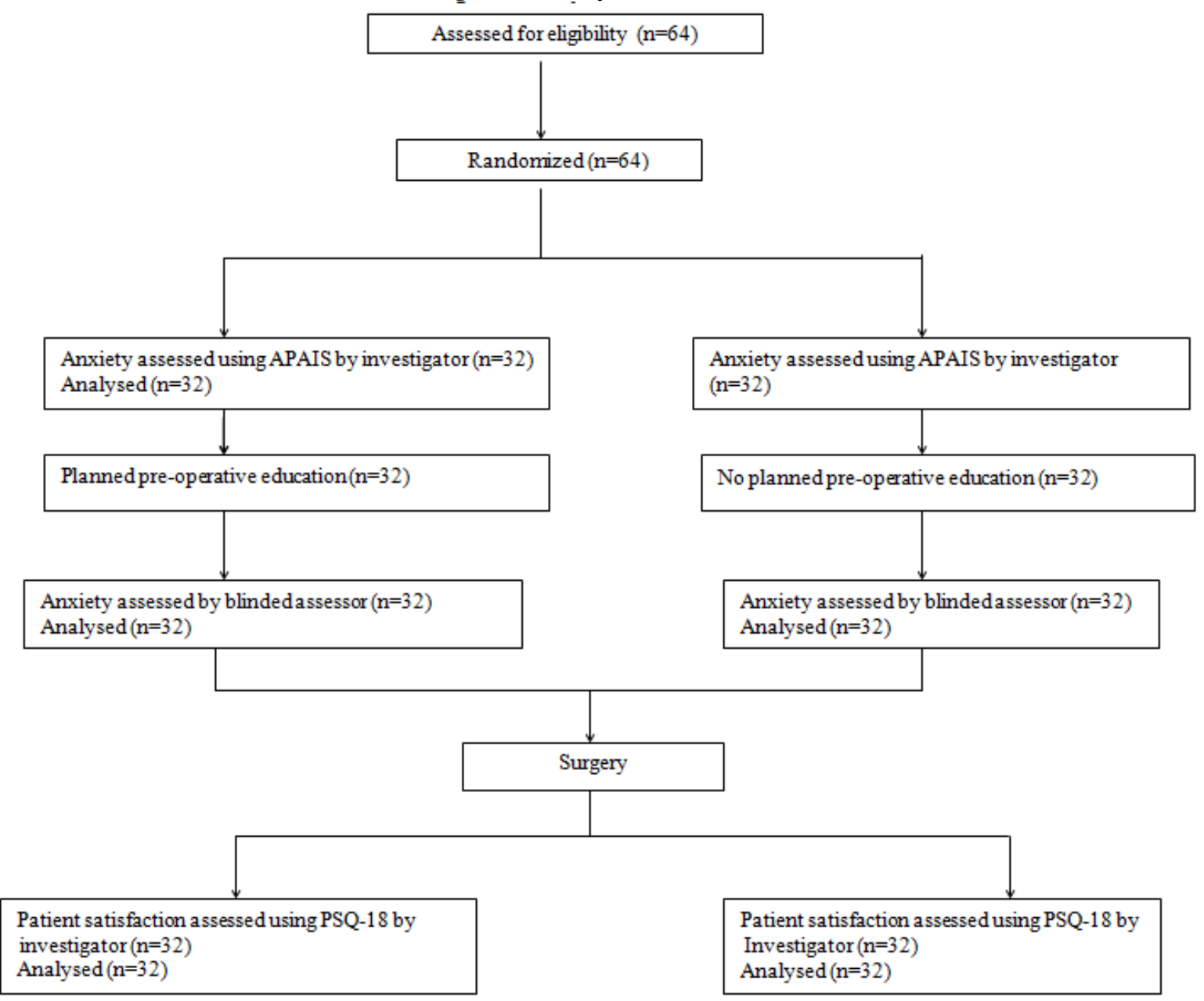

Table 1.Baseline demographic data and baseline results of questionnaires of participants in Group X and Group Y

APAIS-1[Sum C-1]-Combined anxiety component of APAIS assessed by investigator

Sum A-1-Anaesthesia related anxiety

Sum S-1-Surgery related anxiety

Sum I-1-Information desire component.

\begin{tabular}{|lll|}
\hline Demographic data & Group X(n=32) & Group Y(n=32) \\
\hline Age,years[mean \pm SD] & $45.59 \pm 2.781$ & $44.41 \pm 1.829$ \\
\hline Socioeconomic Status [Number (n \%)] & & \\
Low & $13(40.6 \%)$ & $15(46.9 \%)$ \\
Middle & $12(37.5 \%)$ & $15(46.9 \%)$ \\
High & $7(21.9 \%)$ & $2(6.2 \%)$ \\
\hline Educational Status [Number (n \%)] & \\
School & $14(43.8 \%)$ & $15(46.9 \%)$ \\
Graduate & $16(50.0 \%)$ & $17(53.1 \%)$ \\
Post-graduate & $2(6.2 \%)$ & $0(0.0 \%)$ \\
\hline Yes & $9(28.1 \%)$ & $7(21.9 \%)$ \\
No & $23(71.9 \%)$ & $25(78.1 \%)$ \\
\hline APAIS-1[Sum C-1][,mean \pm SD] & $13.25 \pm 4.879$ & $12.28 \pm 5.069$ \\
\hline median,(interquartile range) & & \\
Sum A-1 & $7.00,(5.00-9.00)$ & $6.00,(4.00-8.75)$ \\
Sum S-1 & $7.00,(5.00-9.00)$ & $6.00,(4.00-9.00)$ \\
Sum I-1 & $8.00,(5.00-9.00)$ & $7.00,(4.00-8.00)$ \\
\hline
\end{tabular}


Table2.Outcome Analysis

APAIS-2[Sum C-2]-Combined anxiety component of APAIS assessed by blinded assessor

Sum A-2-Anaesthesia related anxiety

Sum S-2-Surgery related anxiety

Sum I-2-Information desire component.

\begin{tabular}{|c|c|c|c|c|c|}
\hline \multirow[t]{2}{*}{ Item } & \multirow[t]{2}{*}{ Group $X(n=32)$} & \multirow[t]{2}{*}{ Group $Y(n=32)$} & \multirow[t]{2}{*}{$\mathrm{P}$ value } & \multicolumn{2}{|c|}{$\begin{array}{l}95 \% \text { confidence } \\
\text { interval }\end{array}$} \\
\hline & & & & Lower & Upper \\
\hline \multicolumn{6}{|l|}{ APAIS-2[mean $\pm \mathrm{SD}]$} \\
\hline Sum C-2 & $8.06 \pm 4.272$ & $11.84 \pm 5.052$ & 0.002 & -6.122 & -1.441 \\
\hline \multicolumn{6}{|l|}{$\begin{array}{l}\text { APAIS-2 } \\
\text { [median, interquartile range] }\end{array}$} \\
\hline Sum A-2 & $3.00,(2.00-5.00)$ & $6.00,(3.25-8.75)$ & 0.07 & & \\
\hline Sum S-2 & $3.00,(2.00-5.00)$ & $6.00,(4.00-8.75)$ & 0.03 & & \\
\hline Sum I-2 & $7.00,(5.00-7.75)$ & $6.50,(4.25-8.00)$ & 0.724 & & \\
\hline \multicolumn{6}{|l|}{$\begin{array}{l}\text { PSQ-18 } \\
\text { [median, ,interquartile range] }\end{array}$} \\
\hline Overall satisfaction & $69.00,(48.25-75.00)$ & $41.50,(31.00-62.50)$ & $<0.001$ & & \\
\hline General Satisfaction & $3.50,(3.00-4.00)$ & $2.50,(1.62-3.00)$ & 0.001 & & \\
\hline Technical Quality & $3.625,(3.250-4.188)$ & $2.625,(2.000-3.688)$ & $<0.001$ & & \\
\hline Interpersonal Manner & $4.00,(2.00-4.50)$ & $2.00,(2.00-3.00)$ & 0.002 & & \\
\hline Communication & $4.00,(3.00-4.50)$ & $2.50,(1.50-3.50)$ & $<0.001$ & & \\
\hline Financial Aspects & $4.12,(2.38-4.50)$ & $2.00,(1.50-4.00)$ & 0.001 & & \\
\hline Time spent with doctor & $3.75,(2.50-4.50)$ & $2.00,(1.50-3.00)$ & $<0.001$ & & \\
\hline Accessibility and Convenience & $3.5000,(2.3125-4.2500)$ & $2.2500,(1.5000-3.000)$ & $<0.001$ & & \\
\hline
\end{tabular}

